\title{
EL PAPEL DE LA FILOSOFÍA DE LA EDUCACIÓN EN LA FORMACIÓN INICIAL DOCENTE: TENSIONES ENTRE EL DisCuRSO Y LA PRAXIS
}

\author{
Andrés Santa-María ${ }^{2}$ \\ Claudio Tapia Figueroa ${ }^{3}$ \\ Lorena Zuchel ${ }^{4}$
}

\begin{abstract}
Resumen: En este artículo se examina el papel de la enseñanza de la filosofía de la educación en la formación inicial docente, tomando como punto de partida la tensión que es posible notar entre, por un lado, el discurso de la mayoría de las facultades encargadas de preparar profesores, que rescatan la necesidad de formar docentes críticos y reflexivos, y, por otro lado, la praxis de la formación docente que parece tener un acento marcadamente técnico. Ante este escenario, se argumenta para mostrar (1) que la filosofía de la educación sí resulta atingente en el contexto de la formación inicial docente, (2) que lejos de desempeñar una función meramente complementaria en dicha formación, ésta debe cumplir un papel fundamental y, por tanto, articulador de los diversos saberes y competencias propios de un profesor, y (3) que de nada sirve defender el valor fundamental de la filosofía de la educación, si éste no es comprendido como tal por los futuros profesores, por lo que resulta vital pensar cuidadosamente los contenidos, metodologías y momentos adecuados para impartir esta materia.
\end{abstract}

Palabras-clave: Filosofía de la Educación. Formación Inicial Docente. Fundamentos filosóficos. Modelo de Formación basada en competencias.

${ }^{1}$ Este artículo es parte del proyecto interno de investigación PI D 17 01, patrocinado por la Dirección General de Investigación, Innovación y Posgrado de la Universidad Técnica Federico Santa María.

2 Profesor del área de Filosofía del Departamento de Estudios Humanísticos de la Universidad Técnica Federico Santa María, Santiago - Chile. E-mail: andres.santamaria@usm.cl

3 Profesor del área de Historia del Departamento de Estudios Humanísticos de la Universidad Técnica Federico Santa María, Santiago - Chile. E-mail: claudio.tapia@usm.cl

${ }^{4}$ Profesora del área de Filosofía del Departamento de Estudios Humanísticos de la Universidad Técnica Federico Santa María, Santiago - Chile. E-mail: lorena.zuchel@usm.cl

(D)https://orcid.org/0000-0002-4793-595X

http://doi.org/10.1590/0101-3173.2020.v43n2.02.p19 
Oserais-je exposer ici la plus grande, la plus importante, la plus utile règle de toute l'éducation? ce n'est pas de gagner du temps, c'est d'en perdre. Rousseau (1762, p. 202)

\section{Planteo Del Problema}

En su novela 1984, George Orwell (1970, p. 163) introduce el concepto de "doblepensar" para referirse a "[...] la facultad de sostener dos opiniones contradictorias simultáneamente, dos creencias contrarias albergadas a la vez en la mente". Más allá de las implicancias que el uso de este término tiene en la novela, puede resultar útil para describir un rasgo ciertamente peculiar del ser humano. En efecto, no resulta extraño que, en ocasiones, convivan creencias contradictorias en una misma persona, institución o cultura, generando así tensiones a las que usualmente llegamos incluso a acostumbrarnos. Un caso de este tipo de tensiones es la que acontece con respecto al papel que oficialmente se dice asignar y el que realmente se asigna al estudio de las humanidades en el contexto de los proyectos de formación universitaria que pretenden apuntar hacia una 'formación integral'. En lo que respecta a esta comunicación, nos referiremos, en concreto, al problema de la enseñanza de la filosofía de la educación en el contexto de la formación inicial docente para, en primer lugar, destacar la tensión existente entre el discurso institucional que frecuentemente valida dicha enseñanza y la praxis de la docencia que parece desentenderse de ella. A partir de este diagnóstico, defenderemos la tesis de que, de acuerdo con la naturaleza del saber filosófico, la filosofía de la educación no puede cumplir una función meramente complementaria a la formación docente, sino que ésta debe ocupar un lugar necesariamente fundamental. Por último, como una forma de asumir ciertas implicancias de esta tesis, se ensayarán sucintamente algunas ideas que podrían facilitar a la filosofía de la educación entregar todo su potencial formativo y, de esta manera, contribuir a la reducción de esta tensión entre la praxis y el discurso.

Echando un vistazo al panorama actual con respecto a la enseñanza de las humanidades, no se hace difícil ver que estas parecen encontrarse en una franca retirada en la medida en que gana espacio una visión marcadamente pragmática en la esfera política y, consiguientemente, en la pedagógica. A esto se refiere Martha C. Nussbaum (2012, p. 1-11) en su ya célebre ensayo Not for profit. Why democracy needs the humanities, en el que realiza un diagnóstico profundo y preciso de los problemas que hoy enfrenta la educación, con 
especial atención a los efectos sociales que dichos problemas están acarreando. En concreto, Nussbaum se refiere a la crisis educativa actual en términos de "crisis silenciosa". Se trata de una crisis que nace del énfasis exacerbado de las políticas educativas centradas en el 'progreso económico' en desmedro de aquellas que ponen su foco en el 'desarrollo humano'. Es silenciosa por cuanto no se le ha tomado el debido peso, aun cuando sus consecuencias sociales amenazan con ser devastadoras en cuanto al cumplimiento del ideal de una vida en sociedad auténticamente democrática, que debería descansar sobre un sistema de valores cuyo desarrollo sería eminentemente promovido en el contexto del cultivo de las humanidades y las artes, en la medida en que promueve actitudes como la autonomía, el pensamiento crítico, el respeto y la empatía.

Nussbaum propone unos cuantos ejemplos para validar su diagnóstico, en los que recoge algunas experiencias recientes en Estados Unidos e India. Pero no hace falta ir tan lejos para confirmar el advenimiento de la crisis silenciosa. Ya en Chile se generó una bullada polémica en agosto de 2016 a causa de la filtración del documento Reforma educacional en marcha emanado por la Unidad de Currículum y Evaluación del Ministerio de Educación que contenía, entre otras cosas, la propuesta de relegar la asignatura de Filosofía a la condición de materia optativa en los dos últimos ańos de secundaria. Situaciones similares se han dado recientemente en países como Brasil y España. De hecho, si se revisa el panorama a nivel global de la enseñanza de la filosofía a nivel secundario hace poco más de diez años, podemos ver que ya entonces la perspectiva era algo desalentadora (GOUCHA, 2007, 75-92). Por otro lado, si fijamos la mirada en el contexto de la educación superior, es posible notar una actitud cuando menos escéptica por parte de ciertos grupos de estudiantes de aquellas carreras distintas de la licenciatura en Filosofía, como Pedagogía, Derecho o Ingeniería, cada vez que se les exige cursar alguna materia de contenido filosófico como parte de su plan de estudios. Dicha actitud puede ser indicativa del modo en que esta crisis ya se va haciendo presente en el ambiente, al punto de que, no pocas veces, se replica entre aquellas autoridades universitarias que discuten sobre la pertinencia de asignaturas como esta en la formación académica de los estudiantes por cuanto pueden resultar 'poco útiles' para la configuración de las competencias profesionales constitutivas de los perfiles de egreso.

En lo que nos concierne aquí, es decir, en relación con el estatuto actual de la filosofía de la educación en el contexto de los programas de formación 
inicial docente, la crisis silenciosa es fácil de notar. Peter Roberts (2015, p. 627) atribuye este panorama al avance de la ideología neoliberal:

Neoliberalism, whatever form it takes, is strongly instrumentalist in orientation. Philosophy of education is, accordingly, seen by many as 'useless' - as a waste of time given its lack of exchange value in the market (within and beyond the educational sphere). In a world where what counts is that which can be measured (see Biesta 2010) and, in one way or another, sold, philosophy of education is often regarded as of limited value.

El diagnóstico de Roberts se puede ver reforzado si prestamos atención a la adscripción - a menudo acrítica - al programa de Bolonia y al consiguiente modelo de formación por competencias, que redefine la formación universitaria de acuerdo con las necesidades del mercado y del mundo laboral (BICOCCA, 2017, p. 276; ANGULO \& REDÓN, 2011, p. 286-287). Hacia allá parecen apuntar, en efecto, las orientaciones de Perrenoud (2001) para la formación de profesores en sintonía con las necesidades del siglo XXI.

Con todo, el problema del advenimiento de la 'crisis silenciosa' en el contexto de las carreras de pedagogía no parece ser tan simple como una mera retirada de aquellas asignaturas difícilmente articulables con competencias laborales. No se trata, pues, de una suerte de 'retroexcavadora neoliberal' que aplaste, a su paso, todo lo que se aparte de la ideología en boga. El asunto es bastante más sutil por cuanto ha dado lugar a una tensión difícil de interpretar: la tensión entre la praxis y el discurso.

En cuanto a la praxis, nos referimos al ya tantas veces comentado enfoque marcadamente tecnocrático que, a menudo, gobierna la formación inicial docente y, consiguientemente, la pedagogía en el aula (GIROUX, 2001 , p. 62). Este enfoque - que tiende a reducir la práctica docente al ejercicio de una serie de técnicas orientadas a la planificación, evaluación, creación, aplicación de materiales didácticos, etc.- constituye la coronación de la evolución histórica de la identidad profesional docente - tal como muestra Núñez (2007, p. 162) - y que ha cristalizado en una auto-percepción del profesorado como un técnico orientado a la ejecución de programas "a prueba de profesores” (MARTÍN \& PORLÁN, 1999, p. 120). En esta misma línea, el modelo de formación por competencias - presumiblemente útil en el ámbito de la educación técnica-, en la medida en que transpone situaciones propias del ámbito del trabajo al curriculum y la ejecución de la enseñanza centrada en desempeños (NOGUEIRA, 2016, p. 66), tiende a relegar algunos aspectos 
de la formación inicial docente difícilmente medibles pero no por ello menos importantes de cara a los desafíos de la pedagogía en nuestros tiempos, tales como la reflexión, la comprensión o la captación de significados, entre otras disposiciones intelectuales no instrumentales (BICOCCA, 2017, p. 275).

Esta praxis contrasta con el discurso institucional que, en el seno de la mayoría de las facultades que dictan la carrera de pedagogía, tiende a validar la inclusión de al menos una asignatura de filosofía de la educación en los programas de formación inicial docente, siguiendo las orientaciones recomendadas, por ejemplo, por Prieto (2004, p. 46) para que los profesores se conviertan en "agentes activos en la implementación de prácticas pedagógicas transformadoras”. Sólo a modo de ejemplo, se puede ver que, entre las diez facultades chilenas que lideran el ranking 2017 de carreras de Pedagogía Básica de la Revista América Economía (ECHEVERRÍA \& SAEZ 2017), cinco incluyen al menos una asignatura de filosofía de la educación, otras tres incluyen una o más materias de filosofía en general; y las dos restantes tienen al menos alguna asignatura vinculada con teoría de la educación. ${ }^{5} \mathrm{Al}$ menos dos conclusiones pueden extraerse de estos datos: en primer lugar, que no existe un acuerdo unánime acerca de la pertinencia de uno o más cursos sobre filosofía de la educación en el curriculum de las carreras de pedagogía, cosa que no ocurre con otras materias, presumiblemente más 'útiles'. En segundo lugar, lo que podría asomar como un panorama más alentador es que la mayoría de los planes de estudio revisados sí contemplan un espacio para el estudio de la filosofía. Sin embargo, una mirada más profunda permite observar algunos problemas delicados con respecto a la enseñanza de la filosofía de la educación. Entre ellos, que los docentes encargados de dictar la asignatura son, a menudo, profesores o licenciados en Filosofía que poseen una formación filosófica general, pero no en filosofía de la educación como una disciplina de interés en

\footnotetext{
5 De acuerdo con la información disponible al mes de diciembre de 2017 en las páginas web de las universidades incluidas en el ranking, entre las carreras de Pedagogía Básica que tienen alguna asignatura de filosofía de la educación se cuentan las de la U. Católica de Chile ("Educación y filosofía”, séptimo semestre, no obstante se trata de una asignatura electiva), U. Católica de Valparaíso ("Fundamentos filosóficos y sociales de la educación", segundo semestre), la U. de Santiago de Chile ("Filosofía Educacional", segundo semestre), la U. Católica del Maule ("Fundamentos filosóficos y epistemológicos de la educación", primer semestre) y la U. de los Andes ("Filosofía de la Educación" séptimo semestre). Las que tienen alguna asignatura de filosofía en general son la U. Andrés Bello ("Antropología filosófica”, primer semestre), la U. Católica de la Santísima Concepción ("Fundamentos filosóficos", primer semestre, no obstante se trata de una asignatura 'transversal', que no necesariamente está diseñada para la carrera de pedagogía, pues se dicta en todos los planes de estudio que ofrece la universidad) y la U. Alberto Hurtado ("Filosofía social" en el primer semestre y "Filosofía del conocimiento" en el segundo). Por último, las que no imparten asignaturas de filosofía de la educación en particular o de filosofía en general son la U. Diego Portales y la U. de Concepción.
} 
sí misma. Al menos, no se nota, en términos generales, entre las facultades de educación la misma preocupación por contar con docentes-investigadores con una experticia en el tema avalada por posgrados y publicaciones, como sí se ve para el caso de áreas como Curriculum, Evaluación, Planificación, Didácticas, etc. En este sentido, se puede interpretar que, a la hora de constituir equipos de trabajo probadamente competentes para las áreas propias de la formación inicial docente, la filosofía de la educación está lejos de ser una prioridad. Por último, a raíz de estas últimas consideraciones, no resulta extrańo que, al menos a nivel latinoamericano, la creación de una comunidad académica con una agenda de investigación propia en torno a estos temas, como es la Asociación Latinoamericana de Filosofía de la Educación (ALFE), se remonte apenas al año 2010.

Con todo, la tensión entre discurso y praxis se agudiza en la medida en que, a nivel de discurso institucional, la enseñanza de la filosofía de la educación es mayormente validada (al menos está presente en la mayoría de los planes de estudio), pero, a nivel de praxis, la formación y la consiguiente práctica de los profesores discurre al margen de las grandes preguntas filosóficas sobre la educación, como el problema del fin de la educación, los fundamentos epistemológicos del proceso enseñanza-aprendizaje, las dimensiones ética y política de los diversos ámbitos del proceso educativo, etc. Y si el discurso institucional no es capaz de conducir de manera eficaz las grandes tendencias de la Formación Inicial Docente, el resultado final es que ese discurso, en la medida en que expresa un compromiso más teórico que práctico, lejos de aportar a la solución de la crisis silenciosa diagnosticada por Nussbaum (2012), la hace aún más silenciosa.

Frente a este escenario de tensión, y para avanzar en aras de alguna solución, viene al caso problematizar los presupuestos que invisibilizan la crisis mediante el examen de algunas cuestiones de fondo, a saber: ¿Es la filosofía de la educación verdaderamente atingente para la formación docente? Y en el caso de serlo, ¿constituye esta un mero complemento o añadido al proceso de formación inicial docente o constituye, más bien, un elemento clave y articulador de los demás saberes propios del docente? Por último, si desempeńa un papel clave y articulador, surge también la pregunta de en qué momento, en base a qué contenidos y mediante qué metodología debiese impartirse esta materia para que dicho papel clave sea efectivamente percibido como tal por los estudiantes. Estas tres preguntas pueden resumir nuestro planteo del 
problema general del papel de la filosofía de la educación en la Formación Inicial Docente.

\section{El PAPEL DE LA FILOSOFÍA DE LA EDUCACIÓN EN LA FORMACIÓN INICIAL DOCENTE}

Teóricamente, ante estas preguntas cabría la posibilidad de responder admitiendo que la única alternativa es reconocer de una vez que las cosas cambiaron y que en un mundo gobernado por una cosmovisión neoliberal simplemente no hay espacio para cultivar la filosofía de la educación, de modo que reclamar por un lugar en la formación de profesores sería comparable con el alegato que en algunos países ha levantado el gremio de taxistas ante la irrupción de las plataformas Uber y Cabify. Sería entonces el momento de dar un paso al costado y abrazar el nuevo escenario. Esta alternativa puede parecer extrema, y quizá para figurarse sus consecuencias habría que hacer una suerte de ejercicio de ficción, recurso en el que la literatura ha demostrado ser mucho más competente que la filosofía. Un ejemplo claro de ello es el panorama que presenta Ray Bradbury en Farenheit 451, publicada en 1953: una sociedad futura en el que una elite procura el bienestar de la población mediante la prohibición de cualquier actividad - básicamente, el cultivo de las humanidades y de las artes- que distraiga de la obligación de dedicarse a cosas inmediatamente útiles. En medio de esta ficción, vemos al capitán Beatty instruyendo al inocente Guy Montag sobre el sistema educativo en estos términos:

Tranquilidad, Montag. Dale a la gente concursos que puedan ganar recordando la letra de las canciones más populares, o los nombres de las capitales de Estado, o cuánto maíz produjo Iowa el año pasado. Atibórralos de datos no combustibles, lánzales encima tantos «hechos» que se sientan abrumados, pero totalmente al día en cuanto a información. Entonces, tendrán la sensación de que piensan, tendrán la impresión de que se mueven sin estar moviéndose. Y serán felices, porque los hechos de esta naturaleza no cambian. No les des ninguna materia delicada como Filosofía o Sociología para que empiecen a atar cabos. Por ese camino se encuentra la melancolía. Cualquier hombre que pueda desmontar un mural de televisión y volver a armarlo luego - y, en la actualidad, la mayoría de los hombres pueden hacerlo -, es más feliz que cualquier otro que trata de medir, calibrar y sopesar el Universo, que no puede ser medido ni sopesado sin que un hombre se sienta bestial y solitario (BRADBURY, 2006, p. 64. Las cursivas son mías) 
Si para eliminar la tensión entre discurso y praxis la opción es simplemente olvidar lo primero y abrazar acríticamente lo segundo, no estaríamos muy lejos de la distopía de Bradbury. Quizá no haga falta prohibir el estudio de la filosofía; podría bastar con ignorarla. En ese caso, la formación de profesores haría bien en inculcar solamente datos y, sobre todo, técnicas relevantes para la ejecución de la enseñanza. Filosofía o sociología, en cambio, son materias delicadas que generan melancolía o, al menos, perplejidad. Así se encarga de mostrarlo, por ejemplo, Kevin Harris (1999, p. 1), con un elegante toque de ironía, al comienzo de un ensayo sobre los fines de la educación, en el que presenta, en pocas líneas, las enormes diferencias entre los diversos exponentes de la filosofía de la educación a largo de la historia. ${ }^{6}$ Preferible sería entonces la competencia que habilita para montar o desmontar un televisor o, en nuestro caso, planificar una clase o crear un buen instrumento de evaluación.

Lo contrario sería apostar - con los riesgos que suele implicar apostarpor generar perplejidad entre los futuros profesores ante aquellas grandes preguntas que, en cierto modo, exigen pasar por esa disposición afectiva (la perplejidad) antes de ensayar una respuesta. Si el objetivo es que la pedagogía simplemente reproduzca las estructuras sociales, la alternativa más viable es la del comandante Beatty. Si, en cambio, la alternativa más deseable es la de transformar y mejorar dichas estructuras, el cultivo de la filosofía de la educación - con toda la perplejidad o confusión que necesariamente trae consigo- es un camino necesario. Esto es lo que J. Dewey (1993, p. 28-29) denomina el "pensamiento reflexivo", cuya primera fase consiste justamente en "un estado de duda, de vacilación, de perplejidad, de dificultad mental", sin el cual no es posible apuntar al ideal del profesor como intelectual transformativo' que H. Giroux (2001, p. 65) enarbola en varios de sus escritos:

Si creemos que el papel de la enseñanza no puede reducirse al simple adiestramiento en las habilidades prácticas sino que, por el contrario,

\footnotetext{
6 "When students hear that D. H. Lawrence claimed education should aim to 'lead out the individual nature in each man and woman to its true fullness', that for Rousseau the aim of education was 'to come into accord with the teaching of nature', that R. M. Hutchins saw the aim of education as 'cultivation of the intellect', that A. S. Neill believed the aim of education should be to 'make people happier, more secure, less neurotic, less prejudiced', and that John Locke claimed 'education must aim at virtue and teach man to deny his desires, inclinations and appetite, and follow as reason directs'; hopefully the penny has dropped (sc. that 'education' is a changing, contested and often highly personalised, historically and politically constructed concept). Just in case it hasn't I add in that while Pope Pius XI was declaring that the aim of education was to 'cooperate with divine grace in forming the true and perfect Christian', Sergei Shapovalenko insisted that education should aim 'to inculcate the materialist outlook and communist mentality'. That usually does the trick.” (Harris 1999, p. 1)
} 
implica la educación de una clase de intelectuales vital para el desarrollo de una sociedad libre, entonces la categoría de intelectual sirve para relacionar el objetivo de la educación de los profesores, de la instrucción pública y del perfeccionamiento de los docentes con los principios mismos necesarios para desarrollar una ordenación y una sociedad democráticas.

Puestas así las cosas, hay buenas razones para pensar que cabría esperar una diferencia entre un profesor que ha reflexionado seriamente acerca de ciertas cuestiones de filosofía de la educación y uno que no lo ha hecho (HERNÁNDEZ et al. 2015, p. 170). Se podría objetar que el profesor, tal como son sus condiciones laborales, está demasiado ocupado en enseñar como para hacerse cargo de estas cuestiones. Sin embargo, no es menos cierto que esa misma práctica que lo mantiene tan ocupado es la que lo pone frente una serie de preguntas de carácter fundamental a las que urge dar respuesta, aunque sea de un modo provisional, para que esa práctica se desarrolle con un propósito a la vez claro y trascendente. Jacinto Choza (1988, p. 40) dice, con razón, que "el hombre es el único animal que tiene que saber lo que es para serlo”. En efecto, si la educación es, en sí misma, según el decir de Kant (2008, p. 31), una instancia privilegiada en el proceso de humanización del hombre, cualquier proyecto educativo requiere de un esbozo, idea o imagen previa del hombre que permita formular una noción clara de 'persona educada' o 'plena humanidad' (SEVERINO, 2006, p. 621) que oriente, a modo de fin o intención, toda la labor didáctica, de planificación o de evaluación que el profesor debe asumir necesariamente.

Si Hegel (1968, p. 33) tiene razón al decir que la filosofía se ocupa de aquello que es "corrientemente sabido", pero no por ello "conocido", cabe esperar que la filosofía nos enseñe, sobre todo, a mirar, o aún más, a admirar los procesos de enseńanza-aprendizaje y de crecimiento personal no como algo a lo que estamos acostumbrados, sino con la perspectiva ingenua de quien está poco habituado y, por lo mismo, se deja abrazar por la perplejidad y el asombro. Estas disposiciones afectivas no distraen al profesor de su experticia en la materia que enseña y en la didáctica con que la trasmite, sino que le permite distanciarse reflexivamente de ella, pues puede ocurrir $-y$, de hecho, ocurre- que la excesiva preocupación por la práctica obstaculice incluso su buen ejercicio. En síntesis, la reflexión filosófica colabora en la construcción de una identidad profesional docente que se aparta de la visión del profesor operadortecnócrata, en la medida en que le facilita una mayor comprensión de lo que 
hace y para qué lo hace, de modo que actúe con responsabilidad propia, según su modo de comprender e interpretar las situaciones a las que se enfrenta.

A partir de estas reflexiones, podemos contar con algunos elementos para responder a la pregunta de si la filosofía de la educación debe ocupar un papel complementario o fundamental en la formación inicial docente. En atención a la naturaleza propia del saber filosófico - tradicionalmente comprometida con la comprensión de las causas y del sentido de las cosas - difícilmente podría proporcionar todo su potencial formativo si se la concibe como un mero complemento o añadido, a la par de los contenidos y herramientas impartidos en el resto de las asignaturas que configuran el curriculum de una carrera de pedagogía. No se trata, en efecto, de una disciplina que proporcione directamente nuevos conocimientos pedagógicos, sino que se orienta a facilitar una mejor y más profunda comprensión de aquello con lo que un profesor ya está comúnmente familiarizado. El cultivo de la filosofía de la educación no se desarrolla, por tanto, en desmedro, por ejemplo, de la planificación y de la didáctica, sino que, por el contrario, favorece la visibilización y discusión sobre el sentido de la planificación y de la didáctica.

Con todo, este intento por re-pensar la labor educativa desde sus fundamentos filosóficos puede correr el peligro de incurrir en una suerte de auto-idealización de la filosofía de la educación que la vuelva, finalmente, difícil de implementar. Se podría decir, por lo dicho hasta aquí, que la filosofía de la educación debiese ocupar, en teoría o por definición, un papel fundamental, clave y articulador entre los diversos saberes peculiares del profesional de la pedagogía. Sin embargo, no se puede obviar que, en orden a que la filosofía de la educación pueda dar de sí todo lo que se ha sugerido aquí, es necesario pensar también acerca del modo en que esta disciplina debe ser transmitida a los futuros profesores.

\section{FILOSOFÍA DE LA EDUCACIÓN: QUÉ, CÓMO Y CUÁNDO}

Cuando Platón elabora el "curriculum" del filósofo-rey en República VI, ubica muy cuidadosamente las diversas disciplinas según su cronograma del proceso formativo ideal de quienes debiesen gobernar la polis. En efecto, hay un momento para la gimnasia, para las matemáticas, para la dialéctica y, por último, para el conocimiento de la Idea del Bien, que constituye el corazón de la filosofía (JAEGER, 1992, p. 715-723). Más allá de la pertinencia y dudosa factibilidad de dicho curriculum, me parece especialmente rescatable 
la intuición de que existe un tiempo para la filosofía, de modo que, si se la va a incluir en un plan de estudios, su ubicación en el curriculum no puede quedar librada a su suerte, ni se la puede impartir de cualquier manera si la idea es que ella sea comprendida por los estudiantes de acuerdo con el valor que tiene. En relación con la filosofía de la educación, el qué, cómo y cuándo se la enseńe son cuestiones que bien pueden impactar ya sea en la visibilización o invisivilización del papel que ésta debiese cumplir en la formación de los profesores.

La pregunta por el contenido, metodología y momento adecuados para la enseñanza de la filosofía de la educación en las carreras de pedagogía daría para un artículo aparte. Por lo mismo, nos proponemos facilitar aquí solo una reflexión somera y de carácter más bien orientativo acerca de estos asuntos, en orden a reducir la mencionada tensión entre praxis y discurso desde la academia y desde el aula.

El problema no es menor, ya que la filosofía de la educación, como cualquier otra área del saber, abarca mucho más de lo que se puede estudiar en uno o más cursos de pregrado. Matthew Hayden (2012) se encarga de mostrar en un exhaustivo estudio el vastísimo panorama de temas que han sido estudiados en los últimos años bajo el rótulo "filosofía de la educación". Sin embargo, para alivianar la búsqueda de los temas relevantes a estudiar, Stefaan Cuypers (2014, p. 57) traza una distinción metodológicamente luminosa entre la investigación y la enseńanza de esta disciplina. De acuerdo con esta distinción, no resulta raro que la investigación se ocupe de cuestiones eruditas, como suele hacer la scholarship de cualquier área del saber. La enseñanza de la disciplina, en cambio, como cuenta con un tiempo acotado y tiene un propósito propedéutico, debiese ocuparse más bien de cuestiones fundamentales cuidadosamente escogidas. Entre las innumerables propuestas que se puede encontrar para responder a la pregunta de qué enseñar en el poco tiempo del que se dispone, García \& García (2012, p. 31-32), proponen una lista que constituye una buena síntesis de temas especialmente importantes para el educador en cuanto educador (y no en cuanto filósofo de la educación):

- La concepción antropológica que subyace en los distintos modelos teóricos de educación

- Rasgos específicos de la relación educativa

- Relación entre autoridad y libertad 
- Fines, objetivos y valores educativos

- Lugar de la educación cívica, política, ética, religiosa, estética, etc. en el conjunto de un programa educativo

- Deontología profesional docente

Ya con este listado hay para entretenerse bastante. Hernández et al. (2015, p. 172) proponen un elenco de temas bastante similar. Cabe precisar, de todos modos, que especialmente en una asignatura como esta es fundamental privilegiar la calidad por sobre la cantidad, de modo que, ante la necesidad de priorizar un tema particularmente delicado, éste debiese ser el de los fines de la educación. Si Aristóteles (METAFÍSICA I 2) - y luego Tomás de Aquino (SUMMA CONTRA GENTILES I 1), y muchos otros- se refirieron al conocimiento del fin o propósito de las cosas como el elemento más característico del 'oficio del sabio', es porque a partir de ese conocimiento - no por nada llamado "saber arquitectónico"- es posible unificar y orientar adecuadamente todos los demás saberes. Un conocimiento claro de la finalidad última de la actuación educativa permite - como señalan Altarejos \& Naval (2004, p. 85-90) - alcanzar una comprensión de ésta como una unidad en la diversidad de acciones educativas, en la medida en que ese fin último proporciona una "unidad de orden" hacia la cual se dirigen los diversos fines particulares de cada una de dichas acciones. Por eso, el saber acerca del fin de la educación orienta y resignifica los saberes relativos a las numerosas áreas del saber que constituyen el estatuto epistemológico del profesor. Dicho en negativo, olvidado el fin o propósito de la educación, en una época marcada por la tendencia a medirlo todo, no es difícil caer, como advierte Biesta (2010, p. 10-14), en la exacerbación de los diversos datos fácticos que se han erigido como estándares de efectividad, obviando con ello el problema de fondo de los presupuestos que pueden hacer de esa efectividad un valor tan apreciado, toda vez que ésta, bien entendida, constituye un valor instrumental que, como tal, debe estar al servicio de aquellos valores que conforman el fin último de la educación, los cuales son raramente explicitados.

Lo anterior no está exento de dificultades, por cuanto sería, a lo menos, audaz pensar que la discusión filosófica acerca del fin o los fines de la educación se encuentra cerrada. Muy por el contrario, las respuestas ensayadas a lo largo de la historia son variadísimas. Pero como todo buen problema auténticamente filosófico, no podría ser de otra manera. Ya Aristóteles, en el contexto de su 
análisis de los problemas o aporías (literalmente, "pasos obturados") de lo que él llama "filosofía primera", advierte que "la situación aporética de la mente pone de manifiesto lo problemático de la cosa" (METAFÍSICA, 995a30); es decir, si la pregunta por los fines de la educación deja la mente perpleja es porque el asunto en sí mismo ya resulta suficientemente problemático, pero no por ello menos importante. Es la misma idea que formula Dewey (2012, p. 346), cuando plantea que, si existen incertidumbres genuinas en la vida, éstas deberían verse reflejadas en las filosofías.

Esta última reflexión proporciona algunos elementos para pasar a la pregunta de cómo enseñar la filosofía de la educación. Más allá de las cuestiones de carácter didáctico y metodológico, que superarían el propósito de esta comunicación, es importante destacar que, por ejemplo, ante la pregunta sobre los fines de la educación, no se puede cerrar el discurso en una suerte de respuesta canónica. Una actitud auténticamente filosófica sería la que prepara suficientemente la pregunta para que sea el estudiante el que se la plantee. Por muy antigua o clásica que sea una pregunta, se debe saber que ella sólo llega a ser una verdadera pregunta para quien realmente se la hace (TRINDADE 2000, p. 53). Sólo después de ese paso - que coincide con el reconocimiento de la propia ignorancia, que tanto promovía Sócrates en sus interlocutores - es posible buscar respuestas que sean significativas y, consiguientemente, orientativas del resto del quehacer propio del docente. En este sentido, toda auténtica pregunta es, en cierto modo, un comienzo absoluto, un paso obturado que sólo puede ser superado en el encuentro de una respuesta, por tentativa que sea; y esa tarea es, querámoslo o no, indelegable. Esta es, de acuerdo con la lectura de Kohan (2003, p. 225), la fuerza emancipadora y la fuente del valor filosófico-pedagógico de El maestro ignorante de Rancière: la filosofía de la educación es un ejercicio que desacraliza, polemiza e interroga tomando distancia de los modos en que enseñamos y pensamos la educación para, en último término, sumergirnos en un círculo disruptivo de lo obvio, lo normal y lo incuestionado, y del cual sólo podemos salir valiéndonos de nuestra propia inteligencia.

Problemas como la ya mencionada pregunta sobre los fines de la educación, debidamente planteados, es decir, dejando espacio para la perplejidad y para los consiguientes ensayos de respuesta resultan vitales si se considera que, entre los propósitos que debiese perseguir el cultivo de la filosofía de la educación, no sólo se debe contar la adquisición de determinados conocimientos o la fundamentación de ciertas convicciones, sino, antes que 
eso, el ejercicio de aquello a lo que nos referimos más arriba como una actitud auténticamente filosófica, disposición que, en palabras de Dewey (2012, p. 344) prepara para una apertura mental y sensibilidad hacia nuevas percepciones especialmente en relación con la finalidad de la educación, de modo de poder penetrar hacia niveles de significación más profundos y así mirar bajo la superficie de los eventos y objetos en búsqueda de las conexiones que hay entre ellos y comprenderlos no como realidades aisladas sino a la luz de los contextos que constituyen su significación. Esta forma de comprender el ejercicio de la filosofía sienta sus bases en la concepción ancestral de la filosofía como una forma de vida, y que Peter Roberts (2014, p. 632) proyecta a la filosofía de la educación en concreto, entendiéndola como un compromiso hacia un aprendizaje a lo largo de toda la vida, independiente de las condiciones que eventualmente favorezcan o desfavorezcan esta reflexión. Prácticas como el "ensayo de posición pedagógica" que propone Marini (2015, p. 127-130) al modo en que Montaigne concebía el arte de ensayar asoman como un claro ejemplo de actividad que favorece la actitud filosófica, en la medida en que insta al futuro profesor a explicitar, cuestionar $y$, consiguientemente, modificar o fundamentar adecuadamente sus propias convicciones en lo que respecta al arte de la enseñanza.

Por último, un tema que no parece haber sido suficientemente explorado es el de cuándo es más beneficioso el estudio de la filosofía de la Educación en el contexto de los planes de estudios de pedagogía. En la mayoría de los casos, esta materia, justamente por su carácter fundamental, está ubicada entre los primeros dos semestres de carrera (cf. nota 5 acima). Sin embargo, en orden a mantener viva la alianza entre educación y filosofía, resulta al menos tentadora la idea de ubicarla en un punto del proceso formativo que permita una conexión más directa con la práctica educativa, de modo que los problemas filosóficos estudiados no dejen de ser, a la vez, problemas educativos. En uno de los escritos pioneros sobre la relación entre filosofía y formación de profesores, Richard Peters (1967, p. 156) sostiene, con razón a mi juicio, que, entre los criterios que debiesen gobernar la selección de contenidos en un curso de esta naturaleza, debe ocupar un lugar especial el que éstos se vinculen con la práctica.

En consecuencia, quizá lo más adecuado sería contar con más de una asignatura sobre filosofía de la educación, aunque esto podría ser difícil de implementar en el corto plazo. Pero puestos a escoger un momento estratégico para esta asignatura, parece haber mejores razones para pensar en que ésta 
debiese ser impartida preferentemente en la segunda mitad de la carrera, cuando los estudiantes ya han pasado por alguna práctica en aula, cuentan con un bagaje de lecturas capaz de alimentar un discurso, se han planteado preguntas y se han vuelto más difíciles de impresionar que un alumno de primer ańo.

Pero más importante que lo anterior es que, para que este curso no quede sumido a la mera categoría de una suerte de entremés intelectual - en un semestre u otro del plan de estudios-, los problemas estudiados no pueden quedar circunscritos a esta sola asignatura, sino que deberían entrecruzarse con los contenidos de las otras, en incluso discutir con ellos. Es conveniente, en este sentido, que una carrera de pedagogía bien planteada se enmarque en un proyecto educativo integral que se verifique en el trabajo colaborativo y multidisciplinario entre los académicos. Ciertamente, las preguntas y respuestas filosóficas no son estrictamente competencia del experto en didáctica o evaluación, pero eso no exime a esos académicos del deber de abrir el apetito a los estudiantes hacia la grandeza del fenómeno de la educación, más allá incluso de la materia que se está impartiendo. Tan importante como las herramientas que dichas asignaturas entregan a los futuros profesores lo es el que los estudiantes las vean como puertas de acceso a grandes preguntas, para cuya discusión hay al menos una asignatura - Filosofía de la Educación - destinada especialmente, y con la metodología adecuada, para que dichas preguntas sean maduradas, discutidas y, eventualmente, respondidas.

\section{A MODO DE CONCLUSIÓN}

Frente a la tensión comentada aquí entre el discurso institucional que reconoce la importancia de una reflexión transformadora en los profesores y la praxis de la formación docente de carácter más bien tecnocrática, mi propuesta apunta a reducir dicha tensión en favor del discurso, es decir, a tomar acciones para que la praxis se acerque a lo que el discurso pretende. La praxis del trabajo docente se encuentra en constante evolución, la cual ha estado marcada, entre otras cosas, por discursos suficientemente comprometidos como para movilizar a los agentes involucrados en la formación de los futuros profesores. La implementación de metodologías constructivistas o el enfoque educativo centrado en el alumno son algunas de las transformaciones que se han verificado en las últimas generaciones, las cuales han impactado en el ejercicio 
de la docencia a partir de un trabajo formativo sostenido en las facultades de pedagogía, tanto a nivel de la enseñanza como de la investigación.

Puede que la realización del ideal del profesor como un intelectual transformativo' sea uno de los siguientes pasos en esta evolución, lo cual debería verse reflejado en una praxis docente reflexiva y transformadora desde el aula misma. Para eso es indispensable un discurso institucional comprometido, capaz de orientar la formación docente desde sus comienzos. Ya el hecho de que el ideal de un profesor reflexivo esté tan presente en el discurso es un avance, aunque falte aún por asumir sus implicaciones, en orden a reducir esa tensión en favor del discurso, es decir, en orden a transformar la praxis de la formación inicial y, consiguientemente, de la docencia misma, que es lo que más nos interesa. Algunas de esas implicaciones pasan por relativizar aquellos criterios marcadamente pragmáticos que gobiernan, por ejemplo, el modelo de formación basado en competencias, y de ese modo se tome conciencia del papel que cumplen aquellas instancias como la(s) asignatura(s) de filosofía de la educación en la formación de profesores y en la investigación educativa. Sólo a la luz de una mirada amplia y trascendente del rol de los profesores en la sociedad es posible comprender el sentido y la actualidad de las palabras de Rousseau (1762, p. 202) con que comienza este artículo, y aplicarlas específicamente a la formación de los profesores. En efecto, los aprendizajes más difícilmente medibles pero que provocan un mayor impacto en el largo plazo son aquellos que surgen de las instancias que, desde una mirada pragmática, pasarían quizá por una pérdida de tiempo: he ahí la regla más grande y útil de la educación.

Agradecimientos: agradecemos a Silvia Redón por su valiosa orientación en las fases preliminares de esta investigación. Por sus recomendaciones de lecturas deweianas, a Alejandra Olivera. Por sus generosas críticas y observaciones, agradecemos especialmente a Andrea Lizama y Guillermo Marini. Por último, a la Dirección de Investigación de la Pontificia Universidad Católica de Valparaíso por su apoyo en las primeras etapas de este estudio. 
SANTA-MARÍA, A.; FIGUEROA, C. T.; ZUCHEL, L. The role of philosophy of education in the initial teacher training: tensions between the discourse and the Praxis. Trans/form/açâo, Marília, v. 43, n. 2, p. 19-38, Abr./Jun., 2020.

\begin{abstract}
This paper examines the role of the teaching of the philosophy of education in the initial teacher training, taking as a starting point the tension that can be noted between, on the one hand, the discourse of most of the faculties in charge to prepare teachers, who rescue the need to train critical and reflective teachers, and, on the other hand, the praxis of teacher training that seems to have a markedly technical accent. Given this scenario, it is argued to show (1) that the philosophy of education is relevant in the context of initial teacher training, (2) far from playing a purely complementary role in such training, it must play a fundamental role that lets it organize the diverse knowledge and competences of a teacher, and (3) that it is useless to defend the fundamental value of the philosophy of education, if it is not understood as such by prospective teachers, so that it is crucial to think carefully about the contents, methodologies and appropriate moments to impart this subject.
\end{abstract}

Keywords: Philosophy of Education. Initial Teacher Training. Philosophical Foundations. Model of Competency-based learning.

\title{
REFERENCIAS
}

ALTAREJOS, F; NAVAL, C. Filosofía de la Educación. Pamplona: EUNSA, 2006.

ANGULO, F; REDÓN, S. Competencias y contenidos: cada uno es su sitio en la formación docente. Estudios pedagógicos, 37(2), p. 281-299, 2011.

AQUINO, TOMÁS DE. Suma Contra Gentiles. Madrid: BAC. (Trad. y notas L. Robles Carcedo OP y A. Robles Sierra OP), 1967.

ARISTÓTELES. Metafísica. Madrid: Ed. Gredos (Trad. T. Calvo Martínez), 1994.

BICOCCA, R. Análisis crítico-filosófico de las potencialidades educativas de la enseńanza basada en competencias. Educación y Educadores, 20(2), p. 267-281, 2017.

BIESTA, G. Good education in an age of measurement. Boulder, CO: Paradigm, 2010.

BRADBURY, R. Fahrenheit 451. Almería: Ediciones perdidas, 2006.

CHOZA, J. Manual de Antropología Filosófica. Madrid: Rialp, 1988.

CUYPERS, S. The power and limits of philosophy of education. Theory and Research in Education, 12(1), p. 54-64, 2014.

DEWEY, J. Cómo pensamos. Nueva exposición de la relación entre pensamiento y proceso educativo. Barcelona: Paidós, 1993.

DEWEY, J. Democracy and Education. California: Simon \& Brown, 2012. 
ECHEVERRÍA, J; SAEZ, C. Conoce los resultados del Ránking de Universidades Chilenas 2017. Revista América Economía, 2017. Disponible: https://mba. americaeconomia.com/articulos/reportajes/conoce-los-resultados-del-ranking-deuniversidades-chilenas-2017. Consultado el 15-12-2017.

GARCÍA, M; GARCÍA, J. Filosofía de la educación. Cuestiones de hoy y de siempre. Madrid: Narcea, 2012.

GIROUX, H. Los profesores como intelectuales transformativos 1. Revista Docencia 15. p. 60-66, 2001.

GOUCHA, M. La Philosophie, une Ecole de la Liberté. Enseignement de la philosophie et apprentissage du philosopher: état des lieux et regards pour l'avenir. Paris: UNESCO, 2007.

HARRIS, K. Aims! Whose aims? In: MARPLES, R. (ed.) The Aims of Education. London: Routledge, 1999.

HAYDEN, M. What do philosophers of education do? An empirical study of philosophy of education journals. Studies in Philosophy and Education, 31(1), p. 1-27, 2012.

HEGEL, G. La ciencia de la lógica. Buenos Aires: Hachette, 1968.

HERNÁNDEZ, G., CASTILLO, S., ARIAS, L. Construcción de la identidad en el profesional de la educación, a través del curso de filosofía. IXTLI. Revista

Latinoamericana de Filosofía de la Educación, Vol. 2, № 3, p. 165-183, 2015

JAEGER, W. Paideia: los ideales de la cultura griega. México D.F.: Fondo de Cultura Económica, 1992.

KANT, I. Sobre pedagogía. Córdoba: Ed. Universidad Nacional de Córdoba / Encuentro Grupo Editor (Trad. Oscar Caeiro), 2008.

KOHAN, W. O. Três lições de filosofia da educação. Educaçáo \& Sociedade, 24(82), 2003.

MARINI, G. Michel Montaigne como formador de profesores: La necesidad de ensayar la propia posición pedagógica. Trans/Form/Açáo, 38(03), pp 117-132, 2015.

MARTÍN DEL POZO, R; PORLÁN ARIZA, R. Tendencias en la formación inicial del profesorado sobre los contenidos escolares. Revista interuniversitaria de formación del profesorado, (35), p. 115-128, 1999.

NOGUEIRA RAMOS, M.. Políticas educacionais: da pedagogia das competências à pedagogia histórico-crítica. En Barbosa, M.V., Miller, S., Mello S.A. (org.) Teoria histórico-cultural: questóes fundamentais para a educaçáo escolar. São Paulo: Cultura Acadêmica, 1, 59-76, 2016.

NÚNEZ, I. La profesión docente en Chile: Saberes e identidades en su historia. Revista pensamiento Educativo, 41(2), 149-164, 2007.

NUSSBAUM, M. Not for profit: Why democracy needs the humanities. Princeton: Princeton University Press, 2012. 
ORWELL, G. 1984. Madrid: Salvat Editores, 1970.

PERRENOUD, P. La formación de los docentes en el siglo XXI. Revista de Tecnología Educativa, 14(3), p. 503-523, 2001.

PETERS, R. The place of philosophy in the training of teachers, Paedagogica Europaea, 3. p. 152-166, 1967.

PRIETO, M. La construcción de la identidad profesional del docente: un desafío permanente. Enfoques educacionales, 6(1). p. 29-49, 2004.

ROBERTS, P. It was the Best of Times, it was the Worst of Times...: Philosophy of Education in the Contemporary World. Studies in Philosophy and Education, 34(6), p. 623-634, 2015.

ROUSSEAU, J. Emile, ou de l'Éducation, Vol. 1, La Haye: Jean Néaulme, 1762.

SEVERINO, A. J. A busca do sentido da formação humana: tarefa da Filosofia da Educação. Educação e pesquisa, 32(3), p. 619-634, 2006.

TRINDADE, J. A filosofia como actualização da forma do saber (Aristóteles, Metafísica, A1-3), Kleos, 4(4), p. 43-55, 2000.

Recebido: 26/01/2018

Aceito: 15/10/2018 
SANTA-MARÍA, A.; FIGUEROA, C. T.; ZUCHEL, L. 